\title{
A structural basic protein as a counterpart of deoxyribonucleic acid in mammalian spermatozoa
}

It is a well known fact that no nucleohistone, histone or DNA can be extracted from mammalian spermatozoa by the usual methods for extracting these compounds from other cells. This might be explained by supposing that the keratinous membrane, often mentioned in the literature as the cause of the resistance of the heads against mechanical damage, would surround the nucleus. In experiments aimed at the extraction of the above-mentioned compounds we have accordingly taken this possibility into account.

All experiments have been carried out with bovine spermatozoa in the cold, unless stated otherwise.

We extracted complete spermatozoa, freed from plasma, with $0.3 \mathrm{~N} \mathrm{HCl}$ to remove acid-soluble proteins. Evidently the proteins extracted originate from the tails and the thin layer of cytoplasm covering the nucleus, for in electrophoresis at about $\mathrm{pH} 9$ their direction of migration is opposed to that of histones. To detach the tails and middle pieces from the nuclei we then ground the $\mathrm{HCl}$-extracted spermatozoa in a high-speed homogenizer. Upon centrifugation at $550 \times \mathrm{g}$ for about ro min most of the nuclei were sedimented, leaving most of the remaining particulate matter in suspension. A virtually pure preparation of nuclei was obtained by repeatedly suspending the sediment in $0.14 \mathrm{M} \mathrm{NaCl}$ and centrifuging at $55^{\circ} \times \mathrm{g}$, gradually shortening the time of centrifugation a little (see also Dallam And Thomas ${ }^{1}$ ). It was finally washed with water and lyophilized. No protein could be extracted from the dried nuclei by $0.3 \mathrm{~N} \mathrm{HCl}$, indicating that the presumed keratinoid membrane does not become permeable through this treatment. So for extraction of the contents the nuclei would have to be fragmented. We were able to break them up into ro-20 fragments per nucleus by grinding the dry nuclei in a porcelain mortar at room temperature. However, even from these fragments $0.3 \mathrm{~N} \mathrm{HCl}$ or o.I N NaOH did not extract appreciable amounts of protein or DNA. Only with I N NaOH${ }^{*} \mathrm{DNA}$ and protein could be extracted. We studied this method of extraction more extensively, presuming that the proteins thus extracted would be histones. Therefore the amounts of protein were roughly estimated by determining the total amounts of arginine extracted. We found that both the extraction of arginine and of DNA proceed slowly, although in the beginning DNA is extracted much more rapidly than arginine. However, the velocity of the extraction of DNA soon slows down to zero, while the extraction of arginine continues at a constant, very slow rate. After 25 min more than $50 \%$ of the DNA was extracted, against only about $3 \%$ arginine; these percentages increased to about 80 and about Io, respectively, after Ioo min. Even after much longer extraction times a readily sedimenting residue of nuclear fragments remained. We were unable to separate the proteins and the DNA present in the extract by the usual methods for dissociating nucleohistones.

The results of these experiments on broken nuclei suggested to us a second possibility, namely that the nucleus is not enveloped by a keratinoid membrane, but

* The use of I $\mathrm{N} \mathrm{NaOH}$ was suggested to us by a paper by Thomas and Mayer ${ }^{2}$. 
rather the whole nucleus can be regarded as a sponge, consisting of a keratinoid, resembling ordinary keratin in its high sulphur content (the protein of the spermatozoan heads contains about $2.7 \% \mathrm{~S}$, see ref. 3 ), but differing from the latter by a much higher content of arginine (about $35 \%$ in the protein of the heads, and about Io $\%$ in ordinary keratin). DNA and soluble proteins might be enclosed in the cavities of the sponge. The keratinoid material might gradually become more permeable by the increasing damage done to it by the normal $\mathrm{NaOH}$. The arginine extracted could represent destruction products from the keratinoid, but also originally enclosed soluble proteins.

In experiments performed to check this hypothesis the dried nuclei were shown to dissolve readily in performic acid. After dilution with distilled water a highly opalescent solution was obtained, yielding upon centrifugation at $10000 \times \mathrm{g}$ for $30 \mathrm{~min}$ a tough protein pellet, sticking to the wall of the tube. This material, further called Fraction I, also appeared to contain DNA and DNA oxidation products. As the protein is acid-insoluble it is obviously not a classical histone and may represent the remains of the keratinoid in which the sulphur cross linkings have been disrupted by the oxidation with performic acid.

The supernatant was freeze-dried. The dried material dissolved only partly in o. I N HCl. This solution appeared to contain a considerable amount of low-molecularweight degradation products of DNA, which were removed by filtration over a Sephadex G-25 column at room temperature. The protein fraction leaving the column has been called Fraction II.

The fraction of the freeze-dried material, insoluble in $0.1 \mathrm{~N} \mathrm{HCl}$, has been called Fraction III.

The three fractions were analysed together with the solution of dried nuclei in performic acid. Protein was determined by the biuret method; arginine, lysine and histidine according to the STEIN AND MOORE procedure and $\frac{1}{2}$ cystine as cysteic acid according to Schramm et al. ${ }^{4}$, as modified by BANDEMER AND Evans ${ }^{5}$.

The results, assembled in Table $\mathrm{I}$, show that about $70 \%$ of the total protein is present in Fraction I, and that of this protein no less than $35 \%$ is arginine and more than $6 \%$ is $\frac{1}{2}$ cystine, while lysine and histidine are absent. These findings agree very well with our hypothesis that the predominant part of the mass of the nuclei resembles a sponge, consisting of an arginine-rich keratinoid. The Fractions II and III may be mixtures of different composition, possibly containing ordinary sulphur-free soluble histones, sulphur-containing degradation products of the keratinoid, soluble sulphurrich proteins etc. We have calculated from the data in Table I that $70 \%$ of the total

TABLE I

PROTEIN CONTENT OF FRACTIONS AND AMINO ACIDS IN PROTEINS

Protein content expressed relative to amount of protein in performic acid extract of nuclei. Amino acid content expressed as percentage of protein present in the fractions.

\begin{tabular}{lccccc}
\hline & & \multicolumn{3}{c}{ A mino acids in proteins (\%) } \\
\cline { 5 - 6 } & Protein & arg & lys & his & $\frac{1}{2}$ cys \\
\hline Performic acid solution of nuclei & Ioo & 36 & 2.3 & 2.0 & 6.3 \\
Fraction I & 70 & 35 & 0 & 0 & 6.3 \\
Fraction II & I4 & 24 & 2.6 & I.I & 4.6 \\
Fraction III & 7 & I9 & 4.5 & 2.0 & 4.4 \\
\hline
\end{tabular}


arginine of the nuclei is present in Fraction I. If indeed part of the keratinoid has been converted into products found in Fractions II and III, which is not unlikely ${ }^{6}$, the total keratinoid would represent an even higher percentage of the mass of the nuclei, accounting for even more of the total arginine.

The molecular ratio arginine/DNA-phosphoric acid groups in the nuclei has further proved to be about 0.9 , while the molecular ratio total basic amino acids/DNAphosphoric acid groups is only slightly higher, i.e. about I.o. Evidently in bovine spermatozoa the arginine-rich keratinoid fulfils one of the roles (i.e. the binding of DNA-phosphoric acid groups) of the soluble histones in the somatic cells. The DNA will probably be nestled against a network of keratinoid threads. This difference between somatic cells and this representative of mammalian spermatozoa is in line with the view that in the former the histones regulate the function of DNA in protein synthesis, while in spermatozoa DNA has no such function; they only have to transport it in a well protected state.

A detailed report of this investigation will be published.

This work has been supported by grants from the Foundation for Chemical Research in the Netherlands (S.O.N.).

Laboratory for Physiological Chemistry,

The State University,

Elisabeth Bril-Petersen

Utrecht (The Netherlands)

1 R. D. Dallam and L. E. Thomas, Biochim. Biophys. Acta, i I (I953) 79.

2 L. E. Thomas and D. T. Mayer, Science, ito (I949) 393.

3 C. A. Zittle and R. A. O’Delt, J. Biol. Chem., i 40 (I94I) 899.

4 E. Schramm, S. Moore and E. J. Bigwood, Biochem. J., 57 (I954) 33.

5 S. L. Bandemer and R. J. Evans, J. Chromatog., 3 (I960) 43 I.

- J. C. Kendrew, in H. Neurath and K. Bailey, The Proteins, B, Vol. 2, New York, I954, p. 906 .

Received August I5th, I963

Biochim. Biophys. Acta, 76 (I963) I52-I 54

PN 6120

\section{Photosensitized inactivation of deoxyribonucleic acid}

Though it is possible to inactivate transforming DNA with visible light in the presence of a dye ${ }^{1}$, the mechanism of this phenomenon is still unknown. It was recently observed that visible light causes selective destruction of guanine moieties in DNA if methylene blue and oxygen are present ${ }^{2}$. But a complicating factor in most photosensitization experiments is the conversion of the dyes by the light used. For this reason we decided to test lumichrome (I), a compound related to riboflavin and completely stable to visible light.

Transforming DNA, isolated from a Bacillus subtilis wild type strain, was very rapidly inactivated by light of a high-pressure mercury lamp (Philips HP I25) if lumichrome was present. Of the nucleotides occurring_in DNA only deoxyguanylic acid (II) was broken down very quickly under the experimental conditions. The 